\title{
Measurement of Vertical Emittance at LEP from Hard X-Rays
}

\author{
H. Akbari, J. Borer, C. Bovet, Ch. Delmere, A. Manarin, E. Rossa, M. Sillanoli, J. Spanggaard, \\ CERN, SL Division, CH-1211 Geneva
}

\section{Abstract}

Two solid state detectors measure in real time the vertical profiles of both beams in LEP, from hard X-rays produced in normal machine dipoles. Each detector consists of 64 elements of CdTe photoconductor deposited with a pitch of $100 \mu \mathrm{m}$ on a ceramic support. Profiles can be recorded at the rate of bunch passages $(88 \mathrm{kHz})$ for 250 revolutions and are subsequently analysed. This paper describes the new readout electronics adapted to 8 bunch operation of LEP and the performance of detectors using the data acquired in 1992. From the r.m.s. size of these profiles and the knowledge of machine optics, emittance values are obtained.

\section{INTRODUCTION}

The goal of this instrument is to measure, in real time, at a repetition frequency of $88 \mathrm{kHz}$, the vertical emittance of electron and positron bunches in LEP. These particles radiate very large quantity of synchrotron $\mathrm{X}$-ray, in the main dipole magnets [1]. This radiation strikes the detectors, placed in two special recesses [2], through a $0.4 \mathrm{~mm} \mathrm{Be}$ window of the LEP vacuum chamber. There is no imaging of the synchrotron light and the vertical divergence is directly measured by an array of photo conductors (Fig. 1) with a pitch of $100 \mu \mathrm{m}$. Having the knowledge of the machine optics, emittance values can be estimated from the measured profiles [3].

A brief description of the detectors is followed by a presentation of the readout electronics and data acquisition system. The calibration and performance of the detectors are described using the data collected during 1992 LEP Operation. During this period the detector was mainly used to test its performance. All of the results (apart from emittance values) shown here are for the electron bcam. Identical results were obtained for the positron beam.

\section{DETECTORS}

During the normal operation of LEP, where these detectors can be used in parasitic mode permanently, they may be exposed to dose of X-rays (10-100 keV) irradiation up to $10^{12}$ Sv per year. To meet such a requirement, a thin layer $(4 \mu \mathrm{m})$ of polycrystalline CdTe [2] is deposited on ceramic substrate (127 $\mu \mathrm{m}$ thick) which can resist such severe irradiation. The bias line and 64 horizontal finger lines $(50 \mu \mathrm{m}$ wide) with a pitch of $100 \mu \mathrm{m}$, are made with vacuum deposited gold.

During the previous feasibility tests of detectors, they were exposed to an intense $X$-ray radiation ( 6 watts per $\mathrm{mm}^{2}$ ) for a month. This resulted in a deposition of a carbon layer on the photoconductors due to residual gases. This carbon produces a shunt across the photoconductors and increases the dark current. Currently new detectors are being fabricated with a protective $\mathrm{SiO}_{2}$ isolating layer.

Movable mechanics supports the detector mounted on a water cooled aluminium block. It can be displaced vertically within a range of $\pm 10 \mathrm{~mm}$ and rotated for $45^{\circ}$. The movement allows for a proper alignment of the detector with the incident light and also to retract it from irradiation when no data is acquired. The detector is electrically isolated from its support and is contained in a vacuum tank (about $10^{-6}$ torr).

\section{READOUT ELECTRONICS}

In order to obtain the vertical profiles of each LEP beams at every passage of each bunch, the data readout, acquisition and pre-processing must be completed in less than $11 \mu \mathrm{s}$; and the readout must be ready to register the next profile (with 8 bunches per LEP turn). Once the data are readout by the frontend electronics, they must be transferred to the data processors over $300 \mathrm{~m}$ of cable length. Thus to reduce costs (factor 8 about) and fulfil the time requirement for the readout, an analogue signal multiplexing solution has been chosen.

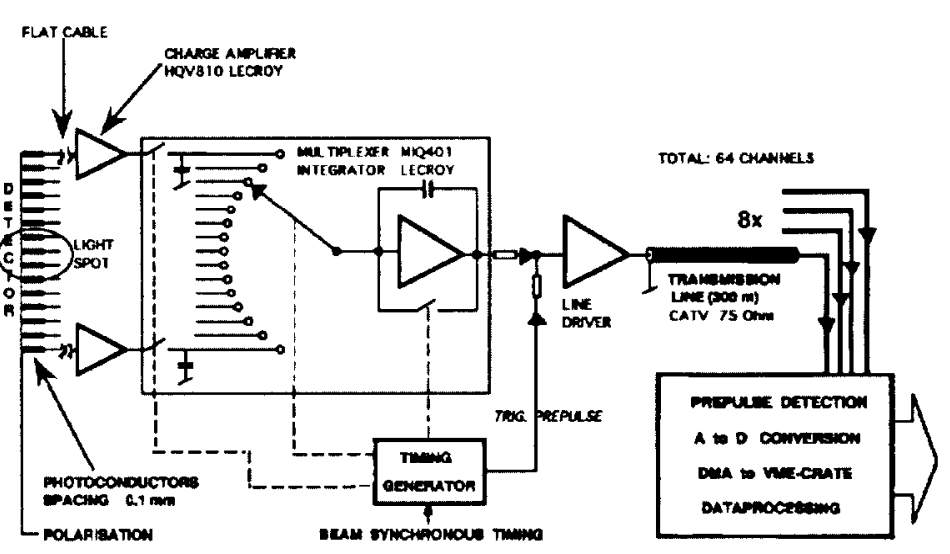

Fig. 1. Synoptic diagram of detector's electronics

Beside the usual difficulties due to mechanical motions in vacuum, a greater technological problem had to be solved: a flat cable with 66 conductors ( $100 \mu \mathrm{m}$ wide and $250 \mu \mathrm{m}$ pitch) and vacuum feed-through to interface the detector with the front-end electronics. The cable is a flexible stripline made with a multilayer of copper and Kapton. An additional external isolating layer avoids parasitic current in the frontend ground layer. The vacuum feed-through is obtained by sealing the flat cable with resin through a slotted flange. This first version was very difficult to connect to the ceramic detector and was fragile. A new version of high mechanical precision, with an intermediate connector and a total length of $2 \mathrm{~m}$, is in preparation. 
The low noise charge-preamplifier integrates for each line the current pulse proportional to the photoconductor illumination. The pulse reflections within the 50 ohms lines of the flat cable have no influence on the total integrated charge since the carrier lifetime in the CdTe is about 10 picoseconds. In such configuration, the photoconductors act as an open circuit and the cable as a capacitive memory. The hybrid circuits, LECROY MIQ401, time multiplexes 64 signals in 8 groups of 8 to form a pulse train within the delay of $11 \mu \mathrm{s}$ between bunches (fig. 1 and 2). A line driver sends the pulses on long distance cables $(300 \mathrm{~m})$ to the data acquisition crate. The time multiplexing is synchronised with turn clock pulses distributed by the Beam Synchronous Timing system which is phase locked to the LEP-RF [4] and delay controlled for selection of bunch.

\section{DATA ACQUISITION SYSTEM}

The data acquisition system is similar to that of the Beam Orbit Measurement system [5]. FADCs are self triggered by the incoming signal. In a sequence they sample first the base line values and second the pulse heights, thus allowing for DC restitution (fig. 2). The data pairs for each channel are stored in a buffer memory with a time sticker. A maximum of 250 beam revolutions can be stored within its depth of 32 kbyte. A secondary memory with smaller depth, $8 \mathrm{kbyte}$, is connected in parallel for other applications like real time observation.

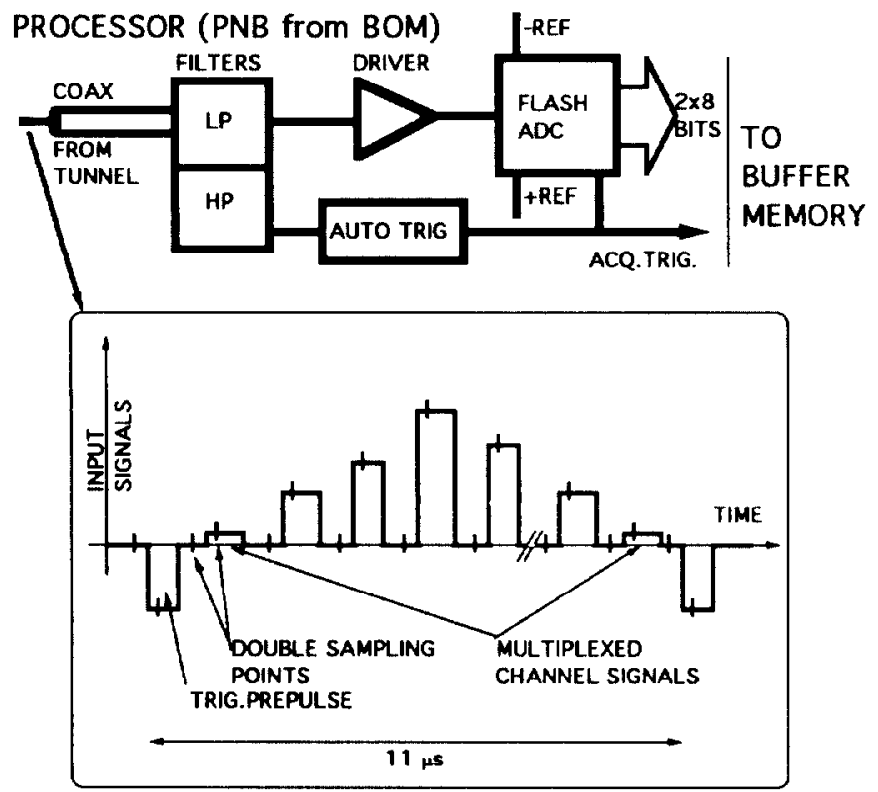

Fig. 2. Acquisition scheme with FADC and buffer memory

The FADCs are connected directly in parallel to the memory module input (VME module) for fast data transfer (about $130 \mathrm{~ns}$ ). The local processor (Device Stub Controller) scans the data and orders them according to their reference position in the detector. The data are normalised with the coefficients obtained from the calibration of the detectors. The result is a file containing the measured vertical profile for each bunch for up to 250 revolutions. This file is then transferred to an Apollo computer for further processing.
The instrument is fully remote controlled: the detector movements, the power supplies, test generators and the analogue direct profile display for maintenance and performance optimisation purposes.

\section{DETECTOR CALIBRATION}

The response of photoconductors can be formulated as following :

$$
R=A_{0}+A_{1} \Phi+A_{2} \Phi V
$$

where $A_{0}$ is the pedestal signal (noise) due to detector and readout electronics in the absence of polarisation voltage $(V)$ and light $(\Phi) ; A_{1} \Phi$ is due to the electrons leaving the surface of photoconductors which are exposed to an intense photon flux but have no polarisation voltage applied; $A_{2} \Phi V$ is the response of the photoconductors to light in presence of voltage.

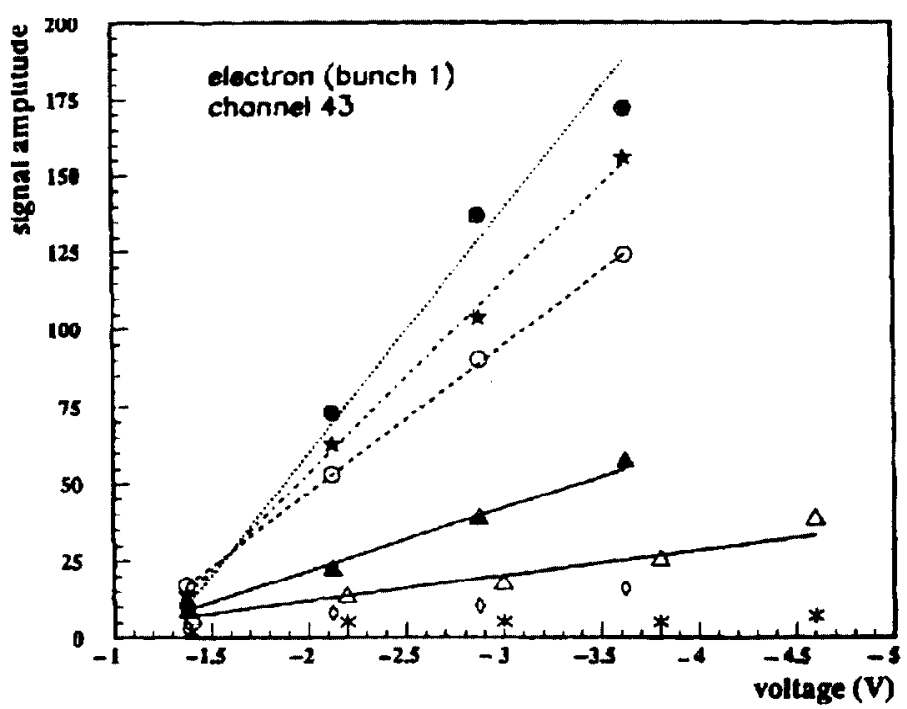

Fig. 3. Response of photoconductor channel 43 as a function of polarisation voltage for different light intensities

The response linearity of each photoconductor channel can be examined as a function of polarization voltage for different intensities of light. All photoconductor channels of both detectors have shown linear response similar to that of channel 43 in fig. 3.

The first step in calibration entails the measurement of $A_{0}$ for all channels when there is no beam. Then a relative calibration of the detector involves the determination of a constant proportional to $A_{1}+A_{2} V$ for each channel as obtained for a given polarization voltage $V$. In order to obtain comparable illumination $(\Phi)$ for each photoconductor, the detector is displaced every $100 \mu \mathrm{m}$ to measure the vertical beam profile at each displacement. In this manner, ideally all photoconductors are exposed to the same light intensity allowing their gains to be compared with each other. Fig. 4 shows a typical measured profile before and after it is calibrated. The results obtained from such calibration are not 
fully satisfactory because of variations observed on some channels due to unstable contact resistance.

\section{PERFORMANCE}

One of the interesting aspects of this detector is its capability to measure the beam profiles for each bunch over 250 turns. The variation of fitted Gaussian parameters for one bunch over six consecutive tums was examined. The mean position varied by $\pm 5 \mu \mathrm{m}$ and the sigma, (typically $\sim 500 \mu \mathrm{m}$ ) by \pm 3 $\mu \mathrm{m})$. These values reflect the precision with which the detector can measure the beam profiles.

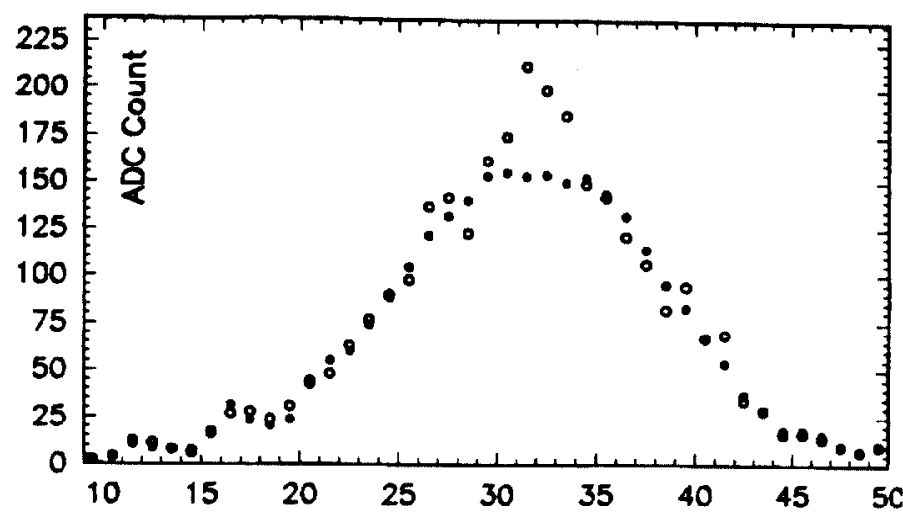

photoconductor chonnel number

Fig. 4. A typical vertical profile before (o) and after $(\bullet)$ calibration

Once the vertical profile of the beam is measured, the vertical emittance can be easily calculated as following :

$$
\varepsilon_{y}=\frac{\sigma_{m}^{2}-\delta_{p h}^{2}}{\beta_{y}}
$$

where $\sigma_{m}$ is the measured sigma, $\delta_{p h}$ is the correction due to photon beam divergence from the light source to the detector and $\beta_{y}$ is the betatron function at the detector. The typical values measured for the sigma of profiles and vertical emittances during 1992 are given below

\begin{tabular}{|l|l|l|}
\hline & $\mathrm{e}^{+}$ & $\mathrm{e}^{-}$ \\
\hline$\sigma_{m}[\mu \mathrm{m}]$ & $\sim 570$ & $\sim 430$ \\
\hline$\varepsilon_{v}[\mathrm{~nm}]$ & $\sim 3.1$ & $\sim 1.5$ \\
\hline
\end{tabular}

During 1992 operation of LEP, the electron and positron beams manifested a $50 \mathrm{~Hz}$ vertical oscillation. Such beam instability has been observed and measured by these detectors [6].

\section{CONCLUSIONS}

With the new electronics vertical profiles can be recorded for the sixteen bunches in LEP, at consecutive revolutions. The signal to noise ratio allows good measurements as from the injection energy of $20 \mathrm{GeV} / \mathrm{c}$. At $45 \mathrm{GeV} / \mathrm{c}$ a filter can be used to select the higher energy part of the X-ray spectrum which allows emittance measurements with an accuracy of $0.1 \mathrm{~nm}$.

More work remains to be done in the hardware stripline connection to the detector and in the software calibration and data analysis programs. Also new photoconductors protected with a thin layer of silicon oxide have to be tested in the beam.

\section{ACKNOWLEDGEMENTS}

We would like to thank M. Cuzin [7] for the supply of CdTe photoconductors and J. Camas, D. Cocq, R. Cornali, B. Hominal, B. Jenny, for the hardware advice and assistance and also the Software Section for the acquisition program.

\section{REFERENCES:}

[1] C. Bovet, J. Kishiro, "A Bcam Profile Monitor using Synchrotron Light", LEP Note 492, 20th March 1984.

[2] E. Rossa, C. Bovet, B. Jenny, J. Spanggaard, E. Jeanclaude, M. Cuzin, M.C. Gentet, C. Ravel, "X-Ray Monitors to Measure Bunch Length and Vertical Profile at LEP" Proc. EPAC Nice (France) 1990.

C. Bovet, E. Rossa, "Measurement of Vertical Emittance at LEP from Hard X-Rays". Workshop on Advance Beam Instrumentation KEK Tsukuba (Japan),22-24 April 1991, Also CERN/SL/91-18(BI).

[4] G. Barribaud et all, "The Beam Synchronous Timing System for the LEP Instrumentation", Int. Conf. on Accelerators and Large Experimental Physics Control Syst., Vancouver, Oct. 30-Nov.3, 1989, Cern/LEP-BI/89-66.

J. Borer,"Instrumentation and Diagnostics Used in LEP Commissioning, with Accent on the LEP Beam Orbit Measurement System", American Inst. of Physics, Conf. proceedings no. 229, Accel. Instrumentation second Annual Workshop, Batavia, Il. 1990.

[6] E. Rossa. "Streak Camera and BEXE". Proc. of Third Workshop on LEP Performance. Chamonix, January 10-16, 1993. Edited by J. Poole. CERN SL/93-19 (DI).

[7] D. LETI, Institut de Recherche Technologique et de Developpement Industriel, C.E.A., 85 X- F.38041 GRENOBLE (FRANCE) 